\title{
Pacific
}

Journal of

Mathematics

\section{MULTIPLICATIVE FUNCTIONS ON FREE GROUPS AND IRREDUCIBLE REPRESENTATIONS}

M. GAbriella Kuhn AND Tim Steger 


\section{MULTIPLICATIVE FUNCTIONS ON FREE GROUPS AND IRREDUCIBLE REPRESENTATIONS}

\section{Gabriella KuhN and Tim Steger}

Let $\Gamma$ be a free group on infinitely many generators. Fix a basis for $\Gamma$ and for any group element $x$, denote by $|x|$ its length with respect to this basis. Let $e$ denote the group identity. A multiplicative function $\phi$ on $\Gamma$ is a function satisfying the conditions $\phi(e)=1$ and $\phi(x y)=\phi(x) \phi(y)$ whenever $|x y|=|x|+|y|$. We characterize those positive definite multiplicative functions for which the associated representation of $\Gamma$ is irreducible.

0. Introduction. Fix an infinite set $A^{+}$and let $\Gamma$ be the free group on generators $A^{+}$. Let $A^{-}$consist of the the inverse generators and let $A=A^{+} \cup A^{-}$. Any $x$ in $\Gamma$ admits a unique shortest expression as a product of elements of $A$. The length of $x,|x|$, is the number of letters in this expression. A multiplicative function $\phi$ on $\Gamma$ is a function satisfying the conditions

$$
\begin{aligned}
\phi(x y) & =\phi(x) \phi(y) \quad \text { when }|x y|=|x|+|y| \\
\phi(e) & =1 .
\end{aligned}
$$

A multiplicative function is determined by its values on $A$.

Choose complex numbers $\{\phi(a)\}_{a \in A}$ such that $\phi\left(a^{-1}\right)=\overline{\phi(a)}$ and satisfying the condition $\sup _{a \in A}|\phi(a)|<1$, and extend $\phi$ to a multiplicative function on $\Gamma$. For example, if $0<r<1$ one can choose $\phi(a)=r$ for every $a$. In that case $\phi(x)=r^{|x|}$ is a radial function which Haagerup [9] showed to be positive definite. DeMichele and Figà-Talamanca [4] extended Haagerup's result, showing that all $\phi$ constructed as above are positive definite.

To each multiplicative positive definite function $\phi$ one associates a unitary representation $\pi_{\phi}$ of $\Gamma$, specified by the property that $\pi_{\phi}$ has a cyclic vector for which $\phi$ is the matrix coefficient. When is $\pi_{\phi}$ 
irreducible? For radial $\phi$ Pytlik and Szwarc [12] demonstrated irreducibility and constructed an explicit realization of $\pi_{\phi}$. Following some of their ideas we prove that the condition

$$
\sum_{a \in A}|\phi(a)|^{2}=+\infty
$$

implies irreducibility.

We show conversely that if the sum $(0.1)$ is finite, then $\pi_{\phi}$ is reducible. If

$$
\sum_{a \in A}|\phi(a)|^{2} /\left(1+|\phi(a)|^{2}\right)
$$

is less than one, then $\phi$ is square integrable and so $\pi_{\phi}$ is a subrepresentation of the regular representation. According to [3] (for all infinite discrete groups) or [10, Section 1] (for the free group), such representations are never irreducible. When $(0.2)$ is equal to one $\phi$ is no longer square integrable, but the associated representation is still expressible as a subrepresentation of the regular representation, and so is still reducible.

Finally, when (0.2) is greater than one, we decompose $\phi$ as the sum of two nonproportional positive definite functions, thereby showing that $\pi_{\phi}$ is reducible. Of the two positive definite summands one is square integrable, and may therefore be further decomposed, while we believe the other to be associated to an irreducible representation. Note that the arguments used in the case $\sum_{a \in A}|\phi(a)|^{2}$ finite may be applied, virtually unchanged, to a free group on only finitely many generators.

1. Construction of $\pi_{\phi}$. From this point on we shall assume that complex numbers $\{\phi(a)\}_{a \in A^{+}}$have been chosen so that

$$
\Phi=\sup _{a \in A^{+}}|\phi(a)|<1 .
$$

Let $\phi$ be the corresponding positive definite function as described in the introduction.

Following the ideas of $[\mathbf{1 2}]$ we proceed to construct a uniformly bounded representation having $\phi$ as a matrix coefficent. For any given $x$ let $x=a_{1} \ldots a_{n-1} a_{n}\left(a_{j} \in A\right)$ be the reduced expression for $x$. Let $\delta_{x}$ denote the Kronecker $\delta$ at the point $x$. Let $\pi_{\text {reg }}$ denote 
the left regular representation of $\Gamma$ on $\ell^{2}(\Gamma)$ so that $\pi_{\text {reg }}(x)\left(\delta_{y}\right)=$ $\delta_{x y}$. Let $H \subset \ell^{2}(\Gamma)$ consist of all finitely supported functions. If $x \in \Gamma$ has reduced expression $a_{1} \ldots a_{n}$, and supposing $x \neq e$, define $\bar{x}=a_{1} \ldots a_{n-1}$. Extend this bar operation to a linear operator on $H$, defining $\overline{\delta_{e}}=0$ and $\overline{\delta_{x}}=\delta_{\bar{x}}$ for $x$ as above. Let $H_{x}$ consist of the functions supported on the set $\{x, \bar{x}, \overline{\bar{x}} \ldots e\}$. If the reader thinks of $\Gamma$ as acting on a homogeneous (locally infinite!) tree, than $H_{x}$ consists of those functions supported on the geodesic from $e$ to $x$. Define a linear operator $P$ on $H$ by

$$
\begin{aligned}
& P\left(\delta_{x}\right)=\phi\left(a_{n}\right) \overline{\delta_{x}} \quad \text { for } x \neq e \\
& P\left(\delta_{e}\right)=0
\end{aligned}
$$

Define a representation $\pi$ of $\Gamma$ on $H$ by

$$
\pi(x)=(I-P)^{-1} \pi_{\text {reg }}(x)(I-P) .
$$

Let $(\cdot, \cdot)$ denote the usual inner product in $\ell^{2}(\Gamma)$.

LEMma 1. The representation $\pi$ extends uniquely to a uniformly bounded representation of $\Gamma$ on $\ell^{2}(\Gamma)$ and $\phi(x)=\left(\pi(x) \delta_{e}, \delta_{e}\right)$.

Proof. Fix $x=a_{1} a_{2} \ldots a_{n}$ in $\Gamma$. Write

$$
\begin{aligned}
\pi(x) \pi_{\mathrm{reg}}\left(x^{-1}\right) & =(I-P)^{-1} \pi_{\mathrm{reg}}(x)(I-P) \pi_{\mathrm{reg}}\left(x^{-1}\right) \\
& =I+\sum_{n=0}^{\infty} P^{n}\left(P-\pi_{\mathrm{reg}}(x) P \pi_{\mathrm{reg}}\left(x^{-1}\right)\right)
\end{aligned}
$$

One may verify that $P-\pi_{\text {reg }}(x) P \pi_{\text {reg }}\left(x^{-1}\right)$ acts as zero on $H_{x}^{\perp}$. Therefore $\pi\left(x^{-1}\right)$ and $\pi_{\text {reg }}\left(x^{-1}\right)$ coincide on $H_{x}^{\perp}$.

Choose $\delta_{e}, \ldots, \delta_{\bar{x}}, \delta_{x}$ as a basis for $H_{x}$. With respect to this basis $P$ acts on $H_{x}$, preserving it, by the following nilpotent upper triangular matrix

$$
\mathbf{P}=\left(\begin{array}{cccc}
0 & \phi\left(a_{1}\right) & \ldots & 0 \\
0 & 0 & \ldots & 0 \\
\vdots & \vdots & \ddots & \phi\left(a_{n}\right) \\
0 & 0 & \ldots & 0
\end{array}\right)
$$

and likewise $\pi_{\text {reg }}(x) P \pi_{\text {reg }}\left(x^{-1}\right)$ is given by the conjugate transpose of $\mathbf{P}$. So the operator norm of $P$ restricted to $H_{x}$ is less than or 
equal to $\Phi$, while the operator norm of $\left(P-\pi_{\mathrm{reg}}(x) P \pi_{\mathrm{reg}}\left(x^{-1}\right)\right)$ will be less than or equal to $2 \Phi$. Therefore, for every $f \in H$ we have

$$
\begin{aligned}
\left\|\pi(x) \pi_{\mathrm{reg}}\left(x^{-1}\right)(f)\right\|_{2} & \leq\|f\|_{2}+2 \Phi \sum_{n=0}^{\infty} \Phi^{n}\|f\|_{2} \\
& \leq\left(\frac{1+\Phi}{1-\Phi}\right)\|f\|_{2}
\end{aligned}
$$

since we have assumed $\Phi<1$. Hence $\pi$ can be extended to a uniformly bounded representation of $\Gamma$. An elementary calculation shows that $\phi(x)=\left(\pi(x) \delta_{e}, \delta_{e}\right)$.

From the proof follows

COROLlaRY. $\pi\left(x^{-1}\right)$ and $\pi_{\text {reg }}\left(x^{-1}\right)$ coincide on $H_{x}^{\perp}$.

Although the representation $\pi$ constructed above is not unitary, it still has some nice properties.

THEOREM 1. Let $\pi$ be as above. Suppose that

$$
\sum_{a \in A}|\phi(a)|^{2}=+\infty
$$

Then $\ell^{2}(\Gamma)$ does not admit any closed linear subspace invariant un$\operatorname{der} \pi$.

Proof. Choose any increasing sequence of subsets $A_{\alpha}$ such that $A_{\alpha}$ is finite for every $\alpha$ and $\sup _{\alpha} \sum_{a \in A_{\alpha}}|\phi(a)|^{2}=+\infty$. Define

$$
U_{\alpha}=\left(\sum_{a \in A_{\alpha}}|\phi(a)|^{2}\right)^{-1} \sum_{a \in A_{\alpha}} \overline{\phi(a)} \pi(a) .
$$

The proof of irreducibility goes as follows:

i) show that $\delta_{e}$ is a cyclic vector for $\pi$.

ii) show that $\left(U_{\alpha} f, g\right)$ converges to $\left(f(e) \delta_{e}, g\right)$ for any $f$ and $g$ in $H$.

iii) show that $U_{\alpha}$ are uniformly bounded as operators from $\ell^{2}(\Gamma)$ into $\ell^{2}(\Gamma)$.

Suppose now that $B$ is any closed invariant linear subspace and let $f \in B$ be a nonzero function. Then by ii) and iii) $U_{\alpha}(f)$ will 
approach $f(e) \delta_{e}$ weakly, showing that $\delta_{e}$ belongs to $B$ provided that $f(e) \neq 0$. If $f(e)=0$ let $x$ be any shortest word in the support of $f$, let $c$ be the last letter of $x$, and use the corollary to Lemma 1 as an aid in calculating

$$
\left(\pi\left(x^{-1}\right) f\right)(e)=\left(1-|\phi(c)|^{2}\right) f(x) \neq 0 .
$$

Then replace $f$ by $\pi\left(x^{-1}\right) f$.

Proof of i). Choose any $x \neq e$ in $\Gamma$. Let $c$ be the last letter of $x$. Then

$$
\delta_{x}=\pi(x) \delta_{e}-\phi(c) \pi(\bar{x}) \delta_{e} .
$$

Proof of ii). First observe that

$$
U_{\alpha}\left(\delta_{e}\right)=\delta_{e}+\left(\sum_{a \in A_{\alpha}}|\phi(a)|^{2}\right)^{-1} \sum_{a \in A_{\alpha}} \overline{\phi(a)} \delta_{a}
$$

and hence $\left(U_{\alpha}\left(\delta_{e}\right), g\right)$ converges to $\left(\delta_{e}, g\right)$ for any $g \in H$. Suppose now $x \neq e$. Then

$$
U_{\alpha}\left(\delta_{x}\right)=\left(\sum_{a \in A_{\alpha}}|\phi(a)|^{2}\right)^{-1}\left(\sum_{a \in A_{\alpha}} \overline{\phi(a)} \delta_{a x}\right)
$$

unless $x^{-1} \in A_{\alpha}$, in which case, for $x=b$, we have

$U_{\alpha}\left(\delta_{b}\right)$

$$
=\left(\sum_{a \in A_{\alpha}}|\phi(a)|^{2}\right)^{-1}\left(\sum_{\substack{a \in A_{\alpha} \\|a b|=2}} \overline{\phi(a)} \delta_{a b}+\overline{\phi\left(b^{-1}\right)}\left(\left(1-|\phi(b)|^{2}\right) \delta_{e}-\overline{\phi\left(b^{-1}\right)} \delta_{b^{-1}}\right)\right)
$$

showing that for every $f$ and $g$ in $H\left(U_{\alpha} f, g\right)$ converges to $\left(f(e) \delta_{e}, g\right)$ $=f(e) \overline{g(e)}$.

Proof of iii). Haagerup's inequality is central for this step. Write

$$
\pi(a)-\pi_{\mathrm{reg}}(a)=\left(\pi(a) \pi_{\mathrm{reg}}\left(a^{-1}\right)-I\right) \pi_{\mathrm{reg}}(a) .
$$

By (1.2)

$$
\left(\pi(a) \pi_{\mathrm{reg}}\left(a^{-1}\right)-I\right)=\sum_{n=0}^{+\infty} P^{n}\left(P-\pi_{\mathrm{reg}}(a) P \pi_{\mathrm{reg}}\left(a^{-1}\right)\right) .
$$


Remember that the right hand side annihilates $H_{a}^{\perp}$. On the other hand, on $H_{a}, P$ and $P-\pi_{\text {reg }}(a) P \pi_{\text {reg }}\left(a^{-1}\right)$ are given respectively by

$$
\left(\begin{array}{cc}
0 & \phi(a) \\
0 & 0
\end{array}\right) \quad \text { and } \quad\left(\begin{array}{cc}
0 & \phi(a) \\
-\overline{\phi(a)} & 0
\end{array}\right)
$$

giving us

$$
\left\|\pi(a) \pi_{\mathrm{reg}}\left(a^{-1}\right)-I\right\|_{2,2} \leq \frac{2|\phi(a)|}{1-\Phi}
$$

and hence

$$
\left\|\pi(a)-\pi_{\mathrm{reg}}(a)\right\|_{2,2} \leq \frac{2|\phi(a)|}{1-\Phi}
$$

Define now

$$
S_{\alpha}=\left(\sum_{a \in A_{\alpha}}|\phi(a)|^{2}\right)^{-1} \sum_{a \in A_{\alpha}} \overline{\phi(a)} \pi_{\mathrm{reg}}(a) .
$$

By Haagerup's inequality (see [9])

$$
\left\|S_{\alpha}\right\|_{2,2} \leq 2\left(\sum_{a \in A_{\alpha}}|\phi(a)|^{2}\right)^{-\frac{1}{2}}
$$

telling us that the $S_{\alpha}$ are uniformly bounded. Finally, by (1.3)

$$
\begin{aligned}
\| U_{\alpha} & -S_{\alpha} \|_{2,2} \\
& =\left(\sum_{a \in A_{\alpha}}|\phi(a)|^{2}\right)^{-1}\left\|\sum_{a \in A_{\alpha}} \overline{\phi(a)}\left(\pi(a)-\pi_{\mathrm{reg}}(a)\right)\right\|_{2,2} \\
& \leq \frac{2}{1-\Phi} .
\end{aligned}
$$

We want to exibit now a unitary irreducible representation having $\phi$ as a matrix coefficent. Let $\left(\pi_{\phi}, \mathcal{H}_{\phi}\right)$ be the representation constructed from $\phi$ by the Gelfand-Raikov construction. Then $\mathcal{H}_{\phi}$ contains a cyclic vector $v_{0}$ with $\left(\pi_{\phi}(x) v_{0}, v_{0}\right) \mathcal{H}_{\phi}=\phi(x)$. One may obtain $\mathcal{H}_{\phi}$ as the completion of the space of finite linear combinations of translates of $v_{0}$ where the square norm of $\sum_{x} c_{x} \pi_{\phi}(x) v_{0}$ is

$$
\left(\sum_{x} c_{x} \pi_{\phi}(x) v_{0}, \sum_{y} c_{y} \pi_{\phi}(y) v_{0}\right)_{\mathcal{H}_{\phi}}=\sum_{x, y \in \Gamma} c_{x} \overline{c_{y}} \phi\left(y^{-1} x\right) \text {. }
$$


We shall prove that $\pi_{\phi}$ is irreducible. Recall that every finitely supported function can be written as a finite linear combination of $\left\{\pi(x)\left(\delta_{e}\right)\right\}_{x \in \Gamma}$. Define first $T: H \subset \ell^{2}(\Gamma) \rightarrow \mathcal{H}_{\phi}$ by letting

$$
T\left(\pi(x) \delta_{e}\right)=\pi_{\phi}(x) v_{0}
$$

and try to extend $T$ to a (nonunitary) linear equivalence from $\ell^{2}(\Gamma)$ to $\mathcal{H}_{\phi}$. If one can do that then $T$ will intertwine $\pi$ to $\pi_{\phi}$ and $\pi_{\phi}$ will also be irreducible.

Observe that at this point, $T$ is not even well defined; nor does one know that the square norm from above, $\left\|\sum_{x} c_{x} \pi_{\phi}(x) v_{0}\right\|_{\mathcal{H}_{\phi}}^{2}=$ $\left(\sum_{x} c_{x} \delta_{x}, \sum_{x} c_{x} \delta_{x}\right)_{\mathcal{H}_{\phi}}$, is comparable with the $\ell^{2}$-square norm of $\sum_{x} c_{x} \pi(x) \delta_{e}$. However, we have

Lemma 2. For every finite linear combination $\sum_{x} c_{x} \pi(x) \delta_{e}$ we have

$$
\left\|\sum_{x} c_{x} \pi_{\phi}(x) v_{0}\right\|_{\mathcal{H}_{\phi}}^{2} \leq\left\|\sum_{x} c_{x} \pi(x) \delta_{e}\right\|_{\ell^{2}(\Gamma)}^{2} \leq \frac{1}{1-\Phi^{2}}\left\|\sum_{x} c_{x} \pi_{\phi}(x) v_{0}\right\|_{\mathcal{H}_{\phi}}^{2}
$$

where $\Phi$ is the constant defined in (1.1).

Proof. Let $\Gamma(y)$ be the set of all words having $y$ as their first piece and $\tilde{\Gamma}(z)$ be the set of all words having $z$ as their last piece. So

$$
\begin{aligned}
& \Gamma(y)=\left\{x \in \Gamma:\left|y^{-1} x\right|=|x|-|y|\right\} \\
& \tilde{\Gamma}(z)=\left\{x \in \Gamma:\left|x z^{-1}\right|=|x|-|z|\right\} .
\end{aligned}
$$

Observe that

$$
\left(\pi(x) \delta_{e}\right)(y)= \begin{cases}0 & x \notin \Gamma(y) \\ \phi\left(y^{-1} x\right) & x \in \Gamma(y)\end{cases}
$$

so that

$$
\left(\sum_{x} c_{x} \pi(x) \delta_{e}\right)(y)=\sum_{x \in \Gamma(y)} c_{x} \phi\left(y^{-1} x\right)
$$

and

$$
\begin{aligned}
\left\|\sum_{x} c_{x} \pi(x) \delta_{e}\right\|_{\ell^{2}(\Gamma)}^{2} & =\sum_{y}\left|\sum_{x \in \Gamma(y)} c_{x} \phi\left(y^{-1} x\right)\right|^{2} \\
& =\sum_{y} \sum_{x, z \in \Gamma(y)} c_{x} \overline{c_{z}} \phi\left(y^{-1} x\right) \overline{\phi\left(y^{-1} z\right)} .
\end{aligned}
$$


For $y \in \Gamma$ let $\Gamma_{2}(y)=\{(x, z): x, z \in \Gamma(y)$ and $x$ and $z$ have exactly $|y|$ initial letters in common $\}$. Then $\Gamma \times \Gamma$ is the disjoint union of the sets $\Gamma_{2}(y)$. Observe that $\phi\left(z^{-1} x\right)=\phi\left(y^{-1} x\right) \overline{\phi\left(y^{-1} z\right)}$ for $(x, z) \in \Gamma_{2}(y)$. Hence

$$
\begin{aligned}
\left\|\sum_{x} c_{x} \pi_{\phi}(x) v_{0}\right\|_{\mathcal{H}_{\phi}}^{2} & =\sum_{x, z} c_{x} \overline{c_{z}} \phi\left(z^{-1} x\right) \\
& =\sum_{y} \sum_{(x, z) \in \Gamma_{2}(y)} c_{x} \overline{c_{z}} \phi\left(y^{-1} x\right) \overline{\phi\left(y^{-1} z\right)} .
\end{aligned}
$$

Subtracting (1.5) from (1.4) we get

$$
\begin{aligned}
\left\|\sum_{x} c_{x} \pi(x) \delta_{e}\right\|_{\ell^{2}(\Gamma)}-\left\|\sum_{x} c_{x} \pi_{\phi}(x) v_{0}\right\| \|_{\mathcal{H}_{\phi}}^{2} \\
=\sum_{y} \sum_{\substack{x, z \in \Gamma(y) \\
(x, z) \notin \Gamma_{2}(y)}} c_{x} \overline{c_{z}} \phi\left(y^{-1} x\right) \overline{\phi\left(y^{-1} z\right)} \\
=\sum_{y} \sum_{\substack{|a|=1 \\
|y a|=|y|+1}} \sum_{x, z \in \Gamma(y a)} c_{x} \overline{c_{z}} \phi\left(y^{-1} x\right) \overline{\phi\left(y^{-1} z\right)} \\
=\sum_{y, a}\left|\sum_{x \in \Gamma(y a)} c_{x} \phi\left(y^{-1} x\right)\right|^{2}
\end{aligned}
$$

that is

$$
\begin{aligned}
0 & \leq\left\|\sum_{x} c_{x} \pi(x) \delta_{e}\right\|_{\ell^{2}(\Gamma)}^{2}-\left\|\sum_{x} c_{x} \pi_{\phi}(x) v_{0}\right\|_{\mathcal{H}_{\phi}}^{2} \\
& =\sum_{y, a}|\phi(a)|^{2}\left|\sum_{x \in \Gamma(y a)} c_{x} \phi\left(a^{-1} y^{-1} x\right)\right|^{2}
\end{aligned}
$$

and letting $y^{\prime}=y a$

$$
\begin{aligned}
& =\sum_{a}|\phi(a)|^{2} \sum_{y^{\prime} \in \tilde{\Gamma}(a)}\left|\sum_{x \in \Gamma\left(y^{\prime}\right)} c_{x} \phi\left(y^{\prime-1} x\right)\right|^{2} \\
& \leq \Phi^{2} \sum_{y^{\prime}}\left|\sum_{x \in \Gamma\left(y^{\prime}\right)} c_{x} \phi\left(y^{\prime-1} x\right)\right|^{2} \leq \Phi^{2}\left\|\sum_{x} c_{x} \pi(x) \delta_{e}\right\|_{\ell^{2}(\Gamma)}^{2} .
\end{aligned}
$$


As a corollary of Lemma 2 we can state

Theorem 2. Suppose that $\sum_{a \in A}|\phi(a)|^{2}=+\infty$ and let $\pi_{\phi}$ be as above. Then $\pi_{\phi}$ is irreducible. Moreover there exists an inner product $(\cdot, \cdot)_{T}$ on $\ell^{2}(\Gamma)$ so that $(\cdot, \cdot)_{T}$ is equivalent to the usual $\ell^{2}$ inner product and the original $\pi$ is unitary with respect to $(\cdot, \cdot)_{T}$.

Proof. Lemma 2 ensures that $T$ extends uniquely to a linear equivalence between $\ell^{2}(\Gamma)$ and $\mathcal{H}_{\phi}$. Since $T$ intertwines $\pi$ to $\pi_{\phi}$, any nontrivial closed invariant subspace of $\mathcal{H}_{\phi}$ comes from a nontrivial closed invariant subspace of $\ell^{2}(\Gamma)$ and the first assertion is a consequence of Theorem 1.

To construct $(\cdot, \cdot)_{T}$ set

$$
(f, g)_{T}=\left(T^{*} T f, g\right)
$$

where $T^{*}$ is the adjoint of $T$ and $(\cdot, \cdot)$ is the $\ell^{2}$ inner product.

$$
\begin{aligned}
(\pi(x) f, \pi(x) g)_{T} & =\left(T^{*} T \pi(x) f, \pi(x) g\right)=(T \pi(x) f, T \pi(x) g)_{\mathcal{H}_{\phi}} \\
& =\left(\pi_{\phi}(x) T f, \pi_{\phi}(x) T g\right)_{\mathcal{H}_{\phi}}=(T f, T g)_{\mathcal{H}_{\phi}} \\
& =\left(T^{*} T f, g\right)=(f, g)_{T} .
\end{aligned}
$$

2. The case $\sum_{a \in A}|\phi(a)|^{2}<+\infty$. Throughout this section we shall assume that $\sum_{a \in A}|\phi(a)|^{2}$ is finite. We observe that all the constructions of the previous section are still valid, but in this case the representation $\pi$ will not be irreducible. Using the arguments of [8, Lemma II.1.5] and the monotonic convergence theorem it is easy to show that $\phi$ will be in $\ell^{2}(\Gamma)$ if and only if $\sum_{a \in A} \frac{|\phi(a)|^{2}}{1+|\phi(a)|^{2}}<1$. Since no $\ell^{2}$ function can be the coefficent of an irreducible representation we are really interested in the case

$$
\sum_{a \in A} \frac{|\phi(a)|^{2}}{1+|\phi(a)|^{2}} \geq 1
$$

Although the case $\sum_{a \in A} \frac{|\phi(a)|^{2}}{1+|\phi(a)|^{2}}=1$ requires more work, we shall see that in that case too $\phi$ is a coefficent of the regular representa- 
tion. First let us consider the opposite case:

$$
\sum_{a \in A} \frac{|\phi(a)|^{2}}{1+|\phi(a)|^{2}}>1 \text {. }
$$

Given (2.1) it is possible to choose a large finite subset $A_{n}$ of $A$ and a positive $\epsilon$ so that

$$
\sum_{a \in A_{n}} \frac{\exp (-2 \epsilon)|\phi(a)|^{2}}{1+\exp (-2 \epsilon)|\phi(a)|^{2}}>1
$$

telling us that the function $\exp (-\epsilon|x|) \phi(x)$ does not belong to $\ell^{2}\left(\Gamma_{n}\right)$ where $\Gamma_{n}$ is the subgroup generated by $A_{n}$. According to Haagerup's result $\phi$ is not a coefficent of a representation weakly contained in the regular. We shall prove the following

Theorem 3. Suppose that (2.1) holds. Then there exists a multiplicative positive definite function $\tilde{\phi}$ and a real number $\kappa>1$ so that $\phi-\frac{\tilde{\phi}}{\kappa}$ is positive definite and $\tilde{\phi}$ is in $\ell^{2}(\Gamma)$.

We were led to this idea by the following facts: when $\phi$ is radial and the set $A$ is finite the representation $\pi$ (to be precise we need to conjugate $\pi$ with a bounded operator to make it unitary) breaks up into two pieces as proved in [14]. One piece is just a subrepresentation of the regular representation and the other gives a representation which is equivalent to one of the complementary spherical series representations of Figà-Talamanca-Picardello [5] as shown in [11]. It is natural to think that the present case will be analogous to the radial case: the spherical series will be replaced by the anisotropic series of Figà-Talamanca-Steger [8]. Since $\phi$ is not weakly contained in the regular representation we expect to see a complementary anisotropic series representation. Both the complementary and the principal anisotropic series can be realized on some suitable space of functions defined on $\Omega$, the boundary of the tree associated with $\Gamma$. Let 1 be the function identically one on $\Omega$. For any $\pi$ in either of the anisotropic series there exists a probability measure $\mu$ on $A$ and a real number $\lambda_{0}$ so that

$$
\pi(\mu) 1=\lambda_{0} 1
$$


The matrix coefficent $\langle\pi(x) 1,1\rangle$ is proportional to the difference of two functions $g_{\pi}$ and $\tilde{g}_{\pi}$ which are each Green functions for $\mu$ and $\lambda_{0}$. That is, $g_{\pi}$ and $\tilde{g}_{\pi}$ both satisfy

$$
g *\left(\lambda_{0} \delta_{e}-\mu\right)=\delta_{e} .
$$

Each of the Green functions is a constant times a multiplicative function.

Keeping this in mind let us return to our case. First we shall look for a measure $\mu$ supported on $A$ and for a real $\lambda_{0}$ so that $\phi$ will satisfy the resolvent condition with respect to $\mu$ and $\lambda_{0}$. Second, we shall look for another function $\tilde{\phi}$ satisfying the same condition. (2.1) will imply that $\tilde{\phi}$ exists and is in $\ell^{2}(\Gamma)$.

Any complex multiplicative $\phi$ can be written as the product of a unitary character $\chi$ with $|\phi|$. If $\phi$ is never zero, then $\chi$ is determined by $\underline{\chi(x)}=\phi(x) /|\phi(x)|$, and is a character because we assume $\phi\left(x^{-1}\right)=\overline{\phi(x)}$. If $\phi(a)=0$ for some $a \in A$, then $\chi(a)$ can be chosen arbitrarily. From this point on we will assume $\phi$ is nonnegative.

First of all we need $\mu$. We want $\mu$ and $\phi$ to satisfy

$$
\begin{aligned}
& \phi * \mu(x)=\lambda_{0} \phi(x) \quad \text { for } x \neq e \\
& \phi * \mu(e)=\lambda_{0} \phi(e)-1
\end{aligned}
$$

for some positive constant $\lambda_{0}$. Letting $x=b(b \in A)$ in (2.2) we get

$$
\mu(b)\left(\phi(b)^{-1}-\phi\left(b^{-1}\right)\right)+\sum_{a \in A} \phi\left(a^{-1}\right) \mu(a)=\lambda_{0} .
$$

Consequently we set

$$
\begin{aligned}
\mu(b) & =\frac{1}{\phi(b)^{-1}-\phi\left(b^{-1}\right)}=\frac{\phi(b)}{1-\phi(b)^{2}} \\
\lambda_{0} & =1+\sum_{a \in A} \frac{\phi(a)^{2}}{1-\phi(a)^{2}} .
\end{aligned}
$$

Since $\phi$ is nonnegative, $\mu$ is a positive measure. If $\mu$ were in $\ell^{1}(\Gamma)$ one might wish to normalize it to be a probability measure. In general, however, $\mu \notin \ell^{1}(\Gamma)$. On the other hand

$$
\sum_{b \in A}|\mu(b)|^{2}=\sum_{b \in A} \frac{\phi(b)^{2}}{\left(1-\phi(b)^{2}\right)^{2}} \leq \frac{1}{\left(1-\Phi^{2}\right)^{2}} \sum_{b \in A} \phi(b)^{2}<+\infty .
$$


As $\mu$ is supported on $A$, Haagerup's inequality says that $\mu$ is a bounded convolver of $\ell^{2}(\Gamma)$. A similar calculation shows that the convolution $\phi * \mu$ exists pointwise.

Look now for another real multiplicative function $\tilde{\phi}$ satisfying $\tilde{\phi}(a)=\tilde{\phi}\left(a^{-1}\right)$ and

$$
\begin{aligned}
& \tilde{\phi} * \mu(x)=\lambda_{0} \tilde{\phi}(x) \quad \text { for } x \neq e \\
& \tilde{\phi} * \mu(e)=\lambda_{0} \tilde{\phi}(e)-\kappa
\end{aligned}
$$

with $\mu$ and $\lambda_{0}$ given by (2.3) and (2.4) and for a suitable constant $\kappa$ to be found. We must solve

$$
\begin{aligned}
\mu(b)\left(\tilde{\phi}(b)^{-1}-\tilde{\phi}\left(b^{-1}\right)\right)+ & \sum_{a \in A} \mu(a) \tilde{\phi}\left(a^{-1}\right)=\lambda_{0} \\
\sum_{a \in A} \mu(a) \tilde{\phi}\left(a^{-1}\right) & =\lambda_{0}-\kappa
\end{aligned}
$$

giving

$$
\kappa=\mu(b)\left(\tilde{\phi}(b)^{-1}-\tilde{\phi}\left(b^{-1}\right)\right)
$$

for all $b \in A$. If such a solution exists, it must be given by

(2.5) $\tilde{\phi}_{\kappa}(a)=\frac{-\kappa\left(\phi(a)^{-1}-\phi(a)\right)+\sqrt{\kappa^{2}\left(\phi(a)^{-1}-\phi(a)\right)^{2}+4}}{2}$

and it must be that

$$
\begin{aligned}
\lambda_{0} & =\kappa+\sum_{a \in A} \mu(a) \tilde{\phi}_{\kappa}(a) \\
& =\kappa+\sum_{a \in A} \frac{1}{2}\left(-\kappa+\sqrt{\kappa^{2}+\frac{4}{\left(\phi(a)^{-1}-\phi(a)\right)^{2}}}\right) \\
& =\kappa+\sum_{a \in A} 2\left(\frac{\phi(a)}{1-\phi(a)^{2}}\right)^{2} \frac{1}{\kappa+\sqrt{\kappa^{2}+\frac{4}{\left(\phi(a)^{-1}-\phi\left(a^{-1}\right)\right)^{2}}}} .
\end{aligned}
$$

If we think of the right hand side of (2.6) as a function $\lambda=\lambda(\kappa)$, we recognize $\lambda(\kappa)$ as the convex function used by Akemann and Ostrand [2] who obtained the convolver norm of $\mu$ as the minimum 
of $\lambda(\kappa)(\kappa>0)$. One may verify that $\lambda(\kappa)$ and $\lambda^{\prime}(\kappa)$ exist for $\kappa>0$. We must now guarantee that $(2.6)$ has a solution for $\lambda_{0}=1+$ $\sum_{a \in A} \frac{\phi(a)^{2}}{1-\phi(a)^{2}}$.

Since this value is attained for $\kappa=1$, since $\lambda(\kappa)$ is continuous, and since $\lambda(\kappa)$ approaches $+\infty$ as $\kappa$ approaches $+\infty$, we only need to check the derivative of $\lambda$ at the point $\kappa=1$. A direct calculation using the identity

$$
\sqrt{\kappa^{2}+\frac{4}{\left(\phi(a)^{-1}-\phi\left(a^{-1}\right)\right)^{2}}}=\left(\tilde{\phi}_{\kappa}(a)+\tilde{\phi}_{\kappa}(a)^{-1}\right) \mu(a)
$$

gives the following formula for $\lambda^{\prime}(\kappa)$ :

$$
\lambda^{\prime}(\kappa)=1-\sum_{a \in A} \frac{\tilde{\phi}_{\kappa}(a)^{2}}{1+\tilde{\phi}_{\kappa}(a)^{2}} .
$$

At $\kappa=1, \tilde{\phi}_{\kappa}=\phi$, so

$$
\lambda^{\prime}(1)=1-\sum_{a \in A} \frac{\phi(a)^{2}}{1+\phi(a)^{2}}<0 .
$$

Hence $\phi$ is the solution of (2.6) which corresponds to a point with negative derivative. Therefore we may be sure that there exists $\kappa_{0}>1$ so that $\lambda\left(\kappa_{0}\right)$ is equal to the value $\lambda_{0}$ given in (2.4).

Define $\kappa_{0}$ to be the unique solution bigger than 1 of (2.6). Let $\tilde{\phi}=\tilde{\phi}_{\kappa_{0}}$ be the multiplicative function defined by (2.5) and see that $\tilde{\phi}$ satisfies $\left(2.2^{\prime}\right)$. By convexity of $\lambda(\kappa)$

$$
\left(1-\sum_{a \in A} \frac{\tilde{\phi}(a)^{2}}{1+\tilde{\phi}(a)^{2}}\right)=\lambda^{\prime}\left(\kappa_{0}\right)>0
$$

and so $\tilde{\phi} \in \ell^{2}(\Gamma)$.

Proof of Theorem 3. Let

$$
\eta=\frac{\kappa_{0} \phi-\tilde{\phi}}{\kappa_{0}-1}
$$

We will show that $\eta$ is positive definite. For $a \in A, \phi(a)=0$ if and only if $\mu(a)=0$ if and only if $\tilde{\phi}(a)=0$. Therefore $\eta$ is supported on the subgroup generated by those $a$ such that $\phi(a) \neq 0$. It is enough 
to prove $\eta$ positive definite on that subgroup. Therefore, passing to the subgroup, we may assume that $\phi(a)$ is never 0 . The proof proceeds in three steps.

Step 1: Construct an algebraic (untopologized) representation $\alpha$ of $\Gamma$ which has $\eta$ as a matrix coefficient.

Step 2: Construct an inner product on the representation space which is preserved by the $\alpha$-action of $\Gamma$.

Step 3: Demonstrate that the inner product is positive semidefinite.

Our algebraic representation will act on a certain subspace $\mathcal{H}^{\infty} \subseteq$ $C(\Omega)$ where $\Omega$ is the boundary of $\Gamma$. Although $\mu$ is not necessarily a finite measure on $A$ and although $\Omega$ is not even locally compact, the main steps of the construction of the complementary series given in [8] are still valid.

Step 1. To construct $\Omega$ think of $\Gamma$ as acting on a homogeneous (locally infinite) tree $\mathcal{T}$. Identify $\Gamma$, as a set, with the set of vertices of $\mathcal{T}$, the action being given by left multiplication. Let $\Omega$ consist of all equivalence classes of half infinite geodesics (two geodesics are said to be equivalent if, up to a shift, they coincide). Any equivalence class has exactly one representative starting at the identity $e$. Denote by $[e, \omega)$ such a geodesic. Choosing $[e, \omega)_{\omega \in \Omega}$ as a set of representatives, we may also identify $\Omega$ with the set of all infinite reduced words. Refer to [7] for unexplained details. Fix $x \in \Gamma$. Let

$$
\Omega(x)=\{\omega \in \Omega:[e, \omega) \text { contains } x\} .
$$

$\Omega(x)$ can also be thought of as the set of all infinite reduced words starting with $x$. Let $\{\Omega(x)\}_{x \in \Gamma}$ constitute a basis for the topology of $\Omega$. Define a probability measure $\nu$ on the sets $\Omega(x)$ by letting

$$
\nu(\Omega(a))=\nu_{a}=\frac{\phi(a) \tilde{\phi}(a)}{1+\phi(a) \tilde{\phi}(a)}
$$

and

$$
\nu(\Omega(x a))=\phi(x) \tilde{\phi}(x) \nu_{a} .
$$

Since $\Omega(x)$ is the disjoint union of the sets $(\Omega(x a))_{|x a|=|x|+1}$ we need

$$
\nu(\Omega(x))=\sum_{\substack{a \in A \\|x a|=|x|+1}} \nu(\Omega(x a)) .
$$


To verify this we only need to show that

$$
\sum_{a \in A} \frac{\phi(a) \tilde{\phi}(a)}{1+\phi(a) \tilde{\phi}(a)}=1
$$

Now

$(2.10)$

$$
\sum_{a \in A} \frac{\phi(a) \tilde{\phi}(a)}{1+\phi(a) \tilde{\phi}(a)}=\sum_{a \in A} \frac{\phi(a)-\tilde{\phi}(a)}{\left(\tilde{\phi}(a)^{-1}-\tilde{\phi}(a)\right)-\left(\phi(a)^{-1}-\phi(a)\right)}
$$

$$
\mu(a)\left(\phi(a)^{-1}-\phi(a)\right)=1 \quad \text { and } \quad \mu(a)\left(\tilde{\phi}(a)^{-1}-\tilde{\phi}(a)\right)=\kappa_{0} .
$$

Applying (2.11) to the right hand side of (2.10) we get

$$
\frac{\sum_{a \in A} \mu(a)(\phi(a)-\tilde{\phi}(a))}{\kappa_{0}-1}=\frac{\left(\lambda_{0}-1\right)-\left(\lambda_{0}-\kappa_{0}\right)}{\kappa_{0}-1}=1
$$

since $\phi$ and $\tilde{\phi}$ satisfy respectively $(2.2)$ and $\left(2.2^{\prime}\right)$. That $\nu$ is a probability measure also follows from (2.9).

If $f(\omega)$ is a bounded complex valued function depending only on the first $n$ letters of $\omega$, define

$$
\int_{\Omega} f(\omega) d \nu(\omega)=\sum_{|x|=n} f(\Omega(x)) \nu(\Omega(x)) .
$$

This is a proper definition in the sense that two values of $n$ lead always to the same value for the integral of $f$. Indeed, one may use (2.8) to verify that $n$ and $n+1$ give the same integral. It is possible, although not necessary in what follows, to extend $\nu$ to the $\sigma$-algebra generated by the sets $\{\Omega(x)\}_{x \in \Gamma}$.

Let 1 denote the function identically one on $\Omega$ and let $1_{x}$ denote the characteristic function of the set $\Omega(x)$. Let $\mathcal{H}^{\infty}$ consist of the linear span of the $\mathbf{1}_{x}$. For $F$ and $G \in \mathcal{H}^{\infty}$, define

$$
\langle F, G\rangle=\int_{\Omega} F(\omega) \overline{G(\omega)} d \nu(\omega) .
$$

For $a \in A$ let

$$
P(a, \omega)= \begin{cases}\phi(a)^{-1} & \text { if } \omega \in \Omega(a) \\ \phi(a) & \text { if } \omega \notin \Omega(a)\end{cases}
$$


Observe that $P\left(a^{-1}, \omega\right) P(a, a \omega)=1$. Since the free group is generated by the $a \in A$ with no relations except $a^{-1} a=e$, it is possible to extend $P(a, \omega)$ to a function $P(x, \omega)$ on $\Gamma \times \Omega$ satisfying the cocycle identity:

$$
P(x y, \omega)=P(x, \omega) P\left(y, x^{-1} \omega\right) .
$$

The outcome is that if $x=a_{1} \ldots a_{n} \in \Gamma$ and $\omega \in \Omega$ have $k$ and only $k$ first letters in common, then

$$
P(x, \omega)=\phi\left(a_{1} \ldots a_{k}\right)^{-1} \phi\left(a_{k+1} \ldots a_{n}\right) .
$$

Observe that $P(x, \omega)$ depends only on the first $|x|$ letters of $\omega$.

For $F \in \mathcal{H}^{\infty}$ define

$$
(\alpha(x) F)(\omega)=P(x, \omega) F\left(x^{-1} \omega\right)
$$

and verify that it gives a representation of $\Gamma$ on $\mathcal{H}^{\infty}$. We claim that

$$
\eta(x)=\langle\alpha(x) 1,1\rangle=\int_{\Omega} P(x, \omega) d \nu .
$$

(2.15) is obviously true when $x=e$. Suppose now that it holds for $|x| \leq n$ and let $y=x a$ with $a \in A$ and $|y|=|x|+1$. By (2.12), the definition of $P(a, \omega)$, and (2.13), the cocycle identity,

$$
P(x a, \omega)= \begin{cases}\phi(a)^{-1} P(x, \omega) & \text { if } \omega \in \Omega(x a) \\ \phi(a) P(x, \omega) & \text { if } \omega \in \Omega \backslash \Omega(x a) .\end{cases}
$$

So

$$
\begin{aligned}
& \int_{\Omega} P(x a, \omega) d \nu \\
& =\phi(a)^{-1} \int_{\Omega(x a)} P(x, \omega) d \nu+\phi(a) \int_{\Omega \backslash \Omega(x a)} P(x, \omega) d \nu \\
& \quad+\phi(a) \int_{\Omega(x a)} P(x, \omega) d \nu-\phi(a) \int_{\Omega(x a)} P(x, \omega) d \nu \\
& =\phi(a) \frac{\kappa_{0} \phi(x)-\tilde{\phi}(x)}{\kappa_{0}-1}+\left(\phi(a)^{-1}-\phi(a)\right) \int_{\Omega(x a)} P(x, \omega) d \nu \\
& =\phi(a) \frac{\kappa_{0} \phi(x)-\tilde{\phi}(x)}{\kappa_{0}-1} \\
& \quad+\left(\phi(a)^{-1}-\phi(a)\right) \phi(x)^{-1} \phi(x) \tilde{\phi}(x) \frac{\phi(a) \tilde{\phi}(a)}{1+\phi(a) \tilde{\phi}(a)} \\
& =\phi(a) \frac{\kappa_{0} \phi(x)-\tilde{\phi}(x)}{\kappa_{0}-1}+\left(\phi(a)^{-1}-\phi(a)\right) \tilde{\phi}(x) \frac{(\phi(a)-\tilde{\phi}(a)) \mu(a)}{\kappa_{0}-1}
\end{aligned}
$$


where for the last equality we wrote $\frac{\phi(a) \tilde{\phi}(a)}{1+\phi(a) \tilde{\phi}(a)}$ as in $(2.10)$ and used (2.11). Therefore

$$
\begin{aligned}
\int_{\Omega} P(x a, \omega) d \nu & =\phi(a) \frac{\kappa_{0} \phi(x)-\tilde{\phi}(x)}{\kappa_{0}-1}+\tilde{\phi}(x) \frac{\phi(a)-\tilde{\phi}(a)}{\kappa_{0}-1} \\
& =\frac{\kappa_{0} \phi(x a)-\tilde{\phi}(x a)}{\kappa_{0}-1}
\end{aligned}
$$

as desired.

Step 2. If $\alpha(x)$ preserved the inner product on $\mathcal{H}^{\infty}$, then (2.15) would imply immediately that $\eta$ was positive definite. Our actual next step is to construct another representation $\tilde{\alpha}$ so that

$$
\langle\alpha(x) F, \tilde{\alpha}(x) G\rangle=\langle F, G\rangle \text {. }
$$

Set

$$
\tilde{P}(a, \omega)= \begin{cases}\tilde{\phi}(a)^{-1} & \text { if } \omega \in \Omega(a) \\ \tilde{\phi}(a) & \text { if } \omega \notin \Omega(a) .\end{cases}
$$

Extend this to a definition of $\tilde{P}(x, \omega)$ using the cocycle identity $(2.13)$. Define

$$
(\tilde{\alpha}(x) F)(\omega)=\tilde{P}(x, \omega) F\left(x^{-1} \omega\right) .
$$

We compute the Radon-Nikodym derivative $d \nu\left(x^{-1} \omega\right) / d \nu(\omega)$ :

$$
\frac{d \nu\left(x^{-1} \omega\right)}{d \nu(\omega)}=P(x, \omega) \tilde{P}(x, \omega)
$$

For $x=a \in A$, this follows from (2.7). To extend to all $x$, use the cocycle identity, which applies to the Radon-Nikodym derivative as well as to $P$ and $\tilde{P}$. Verify $(2.16)$ by

$$
\begin{aligned}
\int_{\Omega} F(\omega) \overline{G(\omega)} d \nu(\omega) & =\int_{\Omega} F\left(x^{-1} \omega\right) \overline{G\left(x^{-1} \omega\right)} P(x, \omega) \tilde{P}(x, \omega) d \nu(\omega) \\
& =\int_{\Omega}(\alpha(x) F)(\omega) \overline{(\tilde{\alpha}(x) G)(\omega)} d \nu(\omega) .
\end{aligned}
$$

From (2.15) follows

$$
\langle\tilde{\alpha}(x) 1,1\rangle=\langle 1, \tilde{\alpha}(x)\rangle^{-}=\left\langle\alpha\left(x^{-1}\right) 1,1\right\rangle^{-}=\bar{\eta}\left(x^{-1}\right)=\eta(x) .
$$


Comparison of $(2.15)$ and $\left(2.15^{\prime}\right)$ suggests the construction of an intertwining map $I$ from $\mathcal{H}^{\infty}$ to $\mathcal{H}^{\infty}$ satisfying

$$
\begin{aligned}
I(1) & =1 \\
I \alpha(x) & =\tilde{\alpha}(x) I .
\end{aligned}
$$

For $|x a|=|x|+1$ a direct computation using (2.11) shows that

$$
\begin{aligned}
\mathbf{1}_{x a} & =\mu(a) \phi(x)(P(x a, \cdot)-\phi(a) P(x, \cdot)) \\
& =\mu(a) \phi(x)(\alpha(x a) \mathbf{1}-\phi(a) \alpha(x) \mathbf{1}) \\
\mathbf{1}_{x a} & =\frac{1}{\kappa_{0}} \mu(a) \tilde{\phi}(x)(\tilde{P}(x a, \cdot)-\tilde{\phi}(a) \tilde{P}(x, \cdot)) \\
& =\frac{1}{\kappa_{0}} \mu(a) \tilde{\phi}(x)(\tilde{\alpha}(x a) \mathbf{1}-\tilde{\phi}(a) \tilde{\alpha}(x) \mathbf{1}) .
\end{aligned}
$$

Therefore 1 is (algebraically) cyclic for $\alpha$ and for $\tilde{\alpha}$. Since we wish $I$ : $\mathcal{H}^{\infty} \rightarrow \mathcal{H}^{\infty}$ to satisfy $(2.17)$ and $(2.18)$, let us try to define it by

$I(\alpha(f) 1)=\tilde{\alpha}(f) \mathbf{1}$ for any finitely supported function $f$ on $\Gamma$.

If $\alpha(f) \mathbf{1}=0$, then for any $x \in \Gamma$

$$
\begin{aligned}
0 & =\left\langle\alpha(f) \mathbf{1}, \tilde{\alpha}\left(\delta_{x}\right) 1\right\rangle=\left\langle\alpha\left(\delta_{x}^{-1} * f\right) 1,1\right\rangle \\
& =\left\langle\tilde{\alpha}\left(\delta_{x}^{-1} * f\right) 1,1\right\rangle=\left\langle\tilde{\alpha}(f) 1, \alpha\left(\delta_{x}\right) 1\right\rangle
\end{aligned}
$$

using the fact that $\langle\alpha(x) 1,1\rangle=\eta(x)=\langle\tilde{\alpha}(x) 1,1\rangle$. Since 1 is cyclic for $\alpha$, it follows that $\tilde{\alpha}(f) \mathbf{1}=0$. Therefore (2.20) is a meaningful definition on its domain. It is also clear that that domain is all of $\mathcal{H}^{\infty}$ and that conditions (2.17) and (2.18) follow from definition (2.20).

Let $f$ and $g$ be two finitely supported functions on $\Gamma$. The following calculation establishes that $I$ is self-adjoint.

$$
\begin{aligned}
\langle\alpha(f) 1, I \alpha(g) 1\rangle & =\langle\alpha(f) 1, \tilde{\alpha}(g) 1\rangle \\
& =\left\langle\alpha\left(g^{*} * f\right) 1,1\right\rangle=\left\langle\tilde{\alpha}\left(g^{*} * f\right) 1,1\right\rangle \\
& =\langle\tilde{\alpha}(f) 1, \alpha(g) 1\rangle=\langle I \alpha(f) 1, \alpha(g) 1\rangle .
\end{aligned}
$$

Define

$$
\langle F, G\rangle_{I}=\langle F, I G\rangle
$$


From (2.16) and (2.18) it follows that $\alpha(x)$ preserves this new inner product. From (2.15) and (2.17) follows $\langle\alpha(x) 1,1\rangle_{I}=\langle\alpha(x) 1,1\rangle=$ $\eta(x)$.

Step 3. It remains to show that $\langle\cdot, \cdot\rangle_{I}$ is positive semidefinite. We proceed to diagonalize $I$. Set

$$
\begin{aligned}
F_{e} & =1 \\
F_{x a} & =\frac{1}{\nu(\Omega(x a))} \mathbf{1}_{x a}-\frac{1}{\nu(\Omega(x))} \mathbf{1}_{x} \quad \text { for }|x a|=|x|+1 .
\end{aligned}
$$

We shall see that each $F_{y}$ is an eigenfunction for $I$.

Consider $F_{x a}$ with $|x|=n$. The integral of $F_{x a}$ over $\Omega(w)$ is zero for any $w$ of length $n$. For any $z$ with $|z| \leq n$ the value of $\tilde{P}(z, \omega)$ depends only on the first $n$ letters of $\omega$. Therefore

$$
\begin{aligned}
0 & =\int_{\Omega} F_{x a}(\omega) \tilde{P}(z, \omega) d \nu(\omega)=\left\langle F_{x a}, \tilde{\alpha}(z) \mathbf{1}\right\rangle \\
& =\left\langle F_{x a}, I \alpha(z) \mathbf{1}\right\rangle=\left\langle I F_{x a}, \alpha(z) \mathbf{1}\right\rangle .
\end{aligned}
$$

Using (2.19), this implies in turn that

$$
0=\left\langle I F_{x a}, 1_{w}\right\rangle=\int_{\Omega(w)} I F_{x a}(\omega) d \nu(\omega)
$$

for any $w$ of length $n$.

Suppose that $n=|x|>0$ and let $x=y b$ where $b$ is the final letter of $x$. According to (2.19) $F_{x a}$ is a linear combination of $\alpha(y) \mathbf{1}, \alpha(x) \mathbf{1}$, and $\alpha(x a) 1$. Consequently $I F_{x a}$ is a linear combination of $\tilde{\alpha}(y) \mathbf{1}$, $\tilde{\alpha}(x) \mathbf{1}$, and $\tilde{\alpha}(x a) \mathbf{1}$, that is $\tilde{P}(y, \cdot), \tilde{P}(x, \cdot)$, and $\tilde{P}(x a, \cdot)$. The form of $\tilde{P}(z, \omega)$ is analogous to that given by $(2.14)$ for $P(z, \omega)$. It follows that $I F_{x a}$ is constant on $\Omega(w)$ for any $w \neq x$ of length $n$, constant on $\Omega(x) \backslash \Omega(x a)$, and constant on $\Omega(x a)$. Together with (2.23), this implies that $I F_{x a}$ equals some multiple of $F_{x a}$. If $n=0, x=e$, an almost identical argument leads to the same conclusion.

For what eigenvalue is $F_{x a}$ an eigenvector?

$$
\left\langle F_{x a}, \alpha(x a) 1\right\rangle=\int_{\Omega} F_{x a}(\omega) P(x a, \omega) d \nu(\omega)
$$




$$
\begin{aligned}
= & \nu(\Omega(x a))\left(\frac{1}{\nu(\Omega(x a))}-\frac{1}{\nu(\Omega(x))}\right) \phi(x)^{-1} \phi(a)^{-1} \\
& -\left(\nu(\Omega(x))-\nu(\Omega(x a)) \frac{1}{\nu(\Omega(x))} \phi(x)^{-1} \phi(a)\right. \\
= & \left(1-\frac{\nu(\Omega(x a))}{\nu(\Omega(x))}\right) \phi(x)^{-1}\left(\phi(a)^{-1}-\phi(a)\right)
\end{aligned}
$$

a direct calculation using (2.14) and (2.22). Similarly

$$
\begin{aligned}
& \left\langle I F_{x a}, \alpha(x a) \mathbf{1}\right\rangle=\left\langle F_{x a}, I \alpha(x a) 1\right\rangle=\left\langle F_{x a}, \tilde{\alpha}(x a) \mathbf{1}\right\rangle \\
& \quad=\left(1-\frac{\nu(\Omega(x a))}{\nu(\Omega(x))}\right) \tilde{\phi}(x)^{-1}\left(\tilde{\phi}(a)^{-1}-\tilde{\phi}(a)\right) .
\end{aligned}
$$

It follows, making use of $(2.11)$, that $I F_{x a}=c_{x} F_{x a}$ with

$$
c_{x}=\frac{\tilde{\phi}(x)^{-1}\left(\tilde{\phi}(a)^{-1}-\tilde{\phi}(a)\right)}{\phi(x)^{-1}\left(\phi(a)^{-1}-\phi(a)\right)}=\kappa_{0} \frac{\phi(x)}{\tilde{\phi}(x)} .
$$

The positivity of the $c_{x}$ gives us what we want. Fix any $G \in \mathcal{H}^{\infty}$. We can write

$$
G=G_{0}+\sum_{x \in \Gamma} G_{x}
$$

where $G_{0}$ is a constant and each $G_{x}$ is a linear combination of the $F_{x a}$ for $a \in A$ such that $|x a|=|x|+1$. Indeed, for $G=1_{z}$ this decomposition is easily accomplished by induction on $|z|$. These terms are orthogonal to one another, and are, moreover, eigenvectors of $I$. Therefore

$$
\langle G, G\rangle_{I}=\langle G, I G\rangle=\left\langle G_{0}, G_{0}\right\rangle+\sum_{x \in \Gamma} c_{x}\left\langle G_{x}, G_{x}\right\rangle \geq 0 .
$$

In [1] Alesina and De Michele showed that under our hypotheses $\phi$ does not belong to the orthogonal complement $A^{\perp}(\Gamma)$ of the Fourier algebra $A(\Gamma)$ in the Fourier-Stieltjes algebra $B(\Gamma)$. Theorem 3 gives more, namely that the representation $\pi_{\phi}$ canonically associated with $\phi$ has a subrepresentation contained in the regular.

THEOREM 4. Suppose that

$$
\sum_{a \in A} \frac{\phi(a)^{2}}{1+\phi(a)^{2}}=1
$$


Then there exists a positive definite $\psi$ in $\ell^{2}(\Gamma)$ so that $\phi=\psi * \psi$.

Proof of Theorem 3. We may, as in the proof of Theorem 2, multiply $\phi$ by a unitary character and so make it nonnegative. Let, as in $(2.4), \lambda_{0}=1+\sum_{a \in A} \frac{\phi(a)^{2}}{1-\phi(a)^{2}}$. For any $\kappa>0$, construct $\tilde{\phi}_{\kappa}$ according to (2.5). Let

$$
\begin{aligned}
\lambda(\kappa) & =\kappa+\sum_{a \in A} \mu(a) \tilde{\phi}_{\kappa}(a) \\
& =\kappa+\sum_{a \in A} \frac{1}{2}\left(-\kappa+\sqrt{\kappa^{2}+\frac{4}{\left(\phi(a)^{-1}-\phi(a)\right)^{2}}}\right)
\end{aligned}
$$

the right hand side of (2.6). Then, by its construction, $\tilde{\phi}_{\kappa}$ satisfies the following version of $\left(2.2^{\prime}\right)$.

$$
\begin{aligned}
& \tilde{\phi}_{\kappa} * \mu(x)=\lambda(\kappa) \tilde{\phi}(x) \quad \text { for } x \neq e \\
& \tilde{\phi}_{\kappa} * \mu(e)=\lambda(\kappa) \tilde{\phi}_{\kappa}(e)-\kappa .
\end{aligned}
$$

As in the proof of Theorem 2 the function $\lambda(\kappa)$ is defined, differentiable, and strictly convex for $\kappa>0$ with derivative

$$
\lambda^{\prime}(\kappa)=1-\sum_{a \in A} \frac{\tilde{\phi}_{\kappa}(a)^{2}}{1+\tilde{\phi}_{\kappa}(a)^{2}} .
$$

This derivative is zero by hypothesis for $\kappa=1$, and so by convexity it is positive for $\kappa>1$. According to Lemmas II.1.5-6 of [8], which extend easily to the case of infinite $A, \sum_{a \in A} \frac{\tilde{\phi}_{\kappa}(a)^{2}}{1+\tilde{\phi}_{\kappa}(a)^{2}}<1$ implies that $\tilde{\phi}_{\kappa}$ is a bounded convolver of $\ell^{2}(\Gamma)$. Equations (2.24) establish then that

$$
\tilde{\phi}_{\kappa}=\kappa(\lambda(\kappa)-\mu)^{-1}
$$

in the algebra of bounded convolvers of $\ell^{2}(\Gamma)$.

Denote by $\operatorname{spec}(\mu)$ the spectrum of $\mu$ as a left convolution operator. By the above $\operatorname{spec}(\mu) \subseteq\left(-\infty, \lambda_{0}\right]$. (It is easy to see but irrelevant to the proof that $\lambda_{0} \in \operatorname{spec}(\mu)$.) Let $E$ be the resolution of the identity for $\mu$ on $\operatorname{spec}(\mu)$. Let $d \sigma=d E_{\delta_{e}, \delta_{e}}$. By the spectral theorem (see Chapter 12 of [13])

$$
\int_{\operatorname{spec}(\mu)} \frac{1}{\lambda(\kappa)-t} d \sigma(t)=\left(\frac{\tilde{\phi}_{\kappa}}{\kappa} * \delta_{e}, \delta_{e}\right)=\frac{\tilde{\phi}_{\kappa}(e)}{\kappa}=\frac{1}{\kappa} \leq 1
$$


for $\kappa>1$. By the monotone convergence theorem

$$
\int_{\operatorname{spec}(\mu)} \frac{1}{\lambda_{0}-t} d \sigma(t) \leq 1
$$

Since $\mu$ acts as a left convolution operator, so does any polynomial in $\mu$ and so does any element of the $C^{*}$-algebra generated by $\mu$. It follows that

$$
d E_{\delta_{x}, \delta_{x}}=d E_{\delta_{e}, \delta_{e}}=d \sigma
$$

for any $x \in \Gamma$. By the Cauchy-Schwarz inequality and the spectral theorem

$$
\begin{aligned}
& \left|\int_{\operatorname{spec}(\mu)} f(t) d E_{\delta_{e}, \delta_{x}}(t)\right| \\
& \quad \leq\left(\int_{\operatorname{spec}(\mu)}|f(t)| d E_{\delta_{e}, \delta_{e}}(t)\right)^{1 / 2}\left(\int_{\operatorname{spec}(\mu)}|f(t)| d E_{\delta_{x}, \delta_{x}}(t)\right)^{1 / 2} \\
& \quad=\int_{\operatorname{spec}(\mu)}|f(t)| d \sigma(t)
\end{aligned}
$$

for any continuous $f(t)$. It follows that $\left|d E_{\delta_{e}, \delta_{x}}\right| \leq d \sigma$. Therefore, using (2.25) and the dominated convergence theorem we may take the limit in

$$
\int_{\operatorname{spec}(\mu)} \frac{1}{\lambda(\kappa)-t} d E_{\delta_{e}, \delta_{x}}(t)=\left(\frac{\tilde{\phi}_{\kappa}}{\kappa} * \delta_{e}, \delta_{x}\right)=\frac{\tilde{\phi}_{\kappa}(x)}{\kappa}
$$

and obtain $\int_{\operatorname{spec}(\mu)} \frac{1}{\lambda_{0}-t} d E_{\delta_{e}, \delta_{x}}(t)=\phi(x)$.

Let

$$
h_{N}(t)= \begin{cases}\left(\lambda_{0}-t\right)^{-1 / 2} & \text { if } \lambda_{0}-t \geq 1 / N \\ 0 & \text { if } \lambda_{0}-t<1 / N .\end{cases}
$$

The spectral theorem establishes that $\psi=\lim _{N \rightarrow \infty} h_{N}(\mu) \delta_{e}$ exists as a limit in $\ell^{2}(\Gamma)$. Moreover

$$
\begin{aligned}
\psi * \psi(x) & =\lim _{N \rightarrow \infty}\left\langle h_{N}^{2}(x) \delta_{e}, \delta_{x}\right\rangle \\
& =\lim _{N \rightarrow \infty} \int_{\operatorname{spec}(\mu) \cap\left\{\lambda_{0}-t \geq 1 / N\right\}} \frac{1}{\lambda_{0}-t} d E_{\delta_{e}, \delta_{x}}(t)=\phi(x) .
\end{aligned}
$$




\section{REFERENCES}

[1] A. Alesina and L. De Michele, A dichotomy for a class of positive definite functions, Pacific J. Math., 103 (1982), 251-257.

[2] C.A. Akemann and P.A. Ostrand, Computing norms in group $C^{*}$-algebras, Amer. J. Math., 98 (1976), 1015-1047.

[3] C. Cecchini and A. Figà-Talamanca, Projections of uniqueness for $L^{p}(G)$, Pacific J. Math., 51 (1974), 37-47.

[4] L. De Michele and A. Figà-Talamanca, Positive definite functions on free groups, Amer. J. Math., 102 (1980), 503-509.

[5] A. Figà-Talamanca and A.M. Picardello, Spherical functions and harmonic analysis on free groups, J. Funct. Anal., 47 (1982), 281-304.

[6] - Harmonic Analysis on Free Groups, Lecture Notes in Pure and Appl. Math., 87, Marcel Dekker, New York, 1983.

[7] A. Figà-Talamanca and C. Nebbia, Harmonic Analysis and Representation Theory for Groups Acting on Homogeneous Trees, London Mathematical Society Lecture Note Series, 162, Cambridge University Press, Cambridge, 1991.

[8] A. Figà-Talamanca and T. Steger Harmonic analysis for anisotropic random walks on homogeneous trees, Mem. Amer. Math. Soc., 531, 215216.

[9] U. Haagerup, An Example of a non nuclear $C^{\star}$-algebra which has the metric approximation property, Invent. Math., 50 (1979), 279-293.

[10] G. W. Mackey, The theory of unitary group representations, Chicago Lectures in Mathematics, The University of Chicago Press Chicago and London, 1976.

[11] A.M. Mantero, T. Pytlik, R. Szwarc and A. Zappa, Equivalence of two series of spherical representations of a free group, Ann. Mat. Pura e Appl., to appear.

[12] T. Pytlik and R. Szwarc, An analytic family of uniformly bounded representations of free groups, Acta Math., 157 (1986), 287-309.

[13] W. Rudin, Functional Analysis, McGraw-Hill, New York, 1973.

[14] R. Szwarc An analytic family of irreducible representations of free group, Annales Inst. Fourier, 38 (1988), 87-110.

Received October 20, 1992 and in revised form January 10, 1994.

Dipartimento di Matematica

Università degli Studi di Milano

Via Saldini 50, 20133 Milano, Italia

E-mail address: kuhn@vmimat.mat.unimi.it 
AND

Istituto di Matematica e Fisica UNIVERSITÀ DEGLI STUdI dI SASSARI Via Vienna 2, 07100 SASSARI, Italia E-mail address: steger@ssmain.uniss.it 


\title{
PACIFIC JOURNAL OF MATHEMATICS
}

Founded by E. F. Beckenbach (1906-1982) and F. Wolf (1904-1989)

\section{EDITORS}

Sun-Yung Alice Chang (Managing Editor)

University of California

Los Angeles, CA 90095-1555

pacific@math.ucla.edu

F. Michael Christ

University of California

Los Angeles, CA 90095-1555

christ@math.ucla.edu

\section{Thomas Enright}

University of California

San Diego, La Jolla, CA 92093

tenright@ucsd.edu

Nicholas Ercolani

University of Arizona

Tucson, AZ 85721

ercolani@math.arizona.edu
Robert Finn

Stanford University

Stanford, CA 94305

finn@gauss.stanford.edu

Vaughan F. R. Jones

University of California

Berkeley, CA 94720

vfr@math.berkeley.edu

Steven Kerckhoff

Stanford University

Stanford, CA 94305

spk@gauss.stanford.edu
Martin Scharlemann

University of California

Santa Barbara, CA 93106 mgscharl@math.ucsb.edu

Gang Tian

Courant Institute

New York University

New York, NY 10012-1100

tiang@taotao.cims.nyu.edu

V. S. Varadarajan

University of California

Los Angeles, CA 90095-1555

vsv@math.ucla.edu

\section{SUPPORTING INSTITUTIONS}

\section{CALIFORNIA INSTITUTE OF TECHNOLOGY NEW MEXICO STATE UNIVERSITY \\ OREGON STATE UNIVERSITY \\ STANFORD UNIVERSITY \\ UNIVERSITY OF ARIZONA \\ UNIVERSITY OF BRITISH COLUMBIA \\ UNIVERSITY OF CALIFORNIA \\ UNIVERSITY OF HAWAII}

\author{
UNIVERSITY OF MONTANA \\ UNIVERSITY OF NEVADA, RENO \\ UNIVERSITY OF OREGON \\ UNIVERSITY OF SOUTHERN CALIFORNIA \\ UNIVERSITY OF UTAH \\ UNIVERSITY OF WASHINGTON \\ WASHINGTON STATE UNIVERSITY
}

The supporting Institutions listed above contribute to the cost of publication of this Journal, but they are not owners or publishers and have no responsibility for its contents or policies.

Manuscripts must be prepared in accordance with the instructions provided on the inside back cover.

The Pacific Journal of Mathematics (ISSN 0030-8730) is published monthly except for July and August. Regular subscription rate: $\$ 215.00$ a year (10 issues). Special rate: $\$ 108.00$ a year to individual members of supporting institutions.

Subscriptions, orders for back issues published within the last three years, and changes of subscribers address should be sent to Pacific Journal of Mathematics, P.O. Box 4163, Berkeley, CA 94704-0163, U.S.A. Prior back issues are obtainable from Kraus Periodicals Co., Route 100, Millwood, NY 10546.

The Pacific Journal of Mathematics at the University of California, c/o Department of Mathematics, 981 Evans Hall Berkeley, CA 94720 (ISSN 0030-8730) is published monthly except for July and August. Second-class postage paid at Berkeley, CA 94704, and additional mailing offices. POSTMASTER: send address changes to Pacific Journal of Mathematics, P.O. Box 6143, Berkeley, CA 94704-0163.

\author{
PUBLISHED BY PACIFIC JOURNAL OF MATHEMATICS at University of California, \\ Berkeley, CA 94720, A NON-PROFIT CORPORATION \\ This publication was typeset using AMS-LATEX, \\ the American Mathematical Society's TEX macro system. \\ Copyright (C) 1995 by Pacific Journal of Mathematics
}




\section{PACIFIC JOURNAL OF MATHEMATICS}

Volume $169 \quad$ No. $2 \quad$ June 1995

On Banach spaces $Y$ for which $B(C(\Omega), Y)=K(C(\Omega), Y)$

201

SHAMIM ISMAIL ANSARI

Convergence of infinite exponentials

219

GENNADY BACHMAN

Cohomologie d'intersection modérée. Un théorème de de Rham

235

Bohumil Cenkl, Gilbert Hector and Martintxo

SARALEGI-ARANGUREN

Kleinian groups with an invariant Jordan curve: $J$-groups

291

Ruben A. Hidalgo

Multiplicative functions on free groups and irreducible representations 311

M. GABRiella KuHN and Tim STEGer

A Diophantine equation concerning finite groups

MAOHUA LE

Nilpotent characters

343

GABRIEL NAVARRO

Smooth extensions and quantized Fréchet algebras

XiaOlU WANG 